\title{
Energy Management of a Microgrid: Compensating for the Difference between the Real and Predicted Output Power of Photovoltaic
}

\author{
Baljit Riar, Member, IEEE, Jaehwa Lee, Alessandra Tosi, Stephen Duncan, Member, IEEE, \\ Michael Osborne, and David Howey, Member, IEEE,
}

\begin{abstract}
An increasing awareness of energy efficiency has led to the development of several improved converter topologies, semiconductor devices and control schemes for distributed energy resources, and, particularly, for microgrids. Recent advances in energy management systems (EMS) for microgrids have improved upon existing methods in several aspects, including prediction of power generated by photovoltaics (PV), and optimal management of electrical energy storage. However, the actual generated PV power may deviate from predictions for several reasons, such as rapid cloud changes or system faults. This paper contributes to the ongoing research on EMS control schemes by proposing a model predictive control (MPC) scheme that adapts to the difference between the actual and predicted output power of PV. The key benefit of this approach is its ability to rapidly adapt to varying operating conditions of the PV without increasing the computational burden of a typical MPC scheme. The feasibility of the scheme is demonstrated using simulations of $5 \mathrm{~kW}$ microgrid system compromising a $5 \mathrm{~kW} / 400$ Ah battery, $10 \mathrm{~kW} P V$ and $5 \mathrm{~kW}$ grid/load connection. The proposed scheme reduces variations in the state of charge (SOC) of a battery. The proposed scheme also reduces the energy taken from grid and this improvement in performance is a function of the difference between the actual and the predicted power.
\end{abstract}

Index Terms-Battery storage system, energy management system, model predictive control, microgrid, optimisation, photovoltaic (PV) system.

\section{INTRODUCTION}

Distributed energy generation (DEG) from renewable energy sources such as solar, wind and hydro is growing in importance in today's power systems. One of the key challenges to scale-up of solar power is the variable, weatherdependent nature of the output. Integration of energy storage systems (ESS) with photovoltaic (PV) arrays is one means of addressing the load management, stability and capacity constraint issues associated with increased roll-out of PV [1]. A microgrid is formed by the integration of DEG, ESS, local loads and a grid connection, and if correctly designed, this may bring a number of benefits over conventional methods of power generation and distribution, such as improved network quality and reduced carbon emissions [2]. There are, however, challenges associated with microgrids, such as reliability issues arising from the variable power generation, optimal scheduling of the ESS charge/discharge to meet demand, control and

Authors are with the Department of Engineering Science, University of Oxford, Oxford, U.K. (emails: baljitriar@ieee.org, jaehwa.lee@eng.ox.ac.uk, alessandra.tosi@eng.ox.ac.uk, stephen.duncan@eng.ox.ac.uk, michael.osborne@eng.ox.ac.uk, and david.howey@eng.ox.ac.uk). protection schemes, and economically optimal operation [3]. This paper focuses on a control scheme for a microgrid where a PV and battery ESS in the $\mathrm{kW}$ power-range are used to support a local grid connection.

Energy management in microgrids is an area of ongoing research, and several control schemes have been proposed to improve performance. In [4], a constrained optimal control problem is solved to minimise variations in the output power of a PV plant. A predictive optimisation algorithm based on dynamic programming has also been used to manage the power output of PV and battery [5]. Model predictive control (MPC) has been widely used with DEG systems because of its ability to handle constraints and to solve the optimisation problem in an intuitive manner. With MPC, a finite horizon open-loop optimal control problem is solved online at each sampling time-step, and its solution is adopted as a control policy until the next time-step [6]. Feedback control is achieved by repeating the optimisation at each step and applying the obtained input until the next time step. A dataenabled model predictive control scheme in combination with an artificial neural network, which predicts the load demand, is used in [7] to improve the efficiency of a small DEG system. For an islanded system, MPC can be combined with weather forecasting models to plan load shedding, thus ensuring critical loads are powered [8]. In [9], MPC is used to minimise both the maintenance and operation cost of a microgrid while accounting for the power consumption and generation from several energy sources.

The actual output power of a PV panel can vary significantly from the predicted output power due to a number of factors, including converter failure, shading of the panels, and accumulation of dirt on the panels. A converter failure results in a sudden reduction in output power, whereas dirt or shading affects the output power more gradually. This paper proposes a variant of a model predictive control scheme to account for the reduction in output power due to the aforementioned factors. The proposed scheme is detailed in section II-C The feasibility of the scheme, which can be adapted easily to different configurations, is demonstrated through simulations of $5 \mathrm{~kW}$ microgrid system and results are presented in section III A comparative investigation with respect to a conventional scheme that does not account for the reduction in output power reveals that the proposed scheme offers superior performance. With a chosen objective of selfconsumption of locally generated power, the energy drawn 
from the grid is used as performance indicator whose value is a function of the discrepancy in the real and predicted output power. With the discrepancy varying in the range of $10 \%$ to $80 \%$, the amount of extra energy given by the grid varies in the range of $0 \%$ to $81 \%$ relative to the case of $10 \%$ discrepancy.

\section{EnERgy MANAGEMENT System}

\section{A. Problem Statement}

The objective of an energy management system (EMS) may be defined in various ways, but a common goal is to minimise the energy taken from the grid (i.e. to maximise 'self-consumption' of locally generated power). For several energy sources, a balance equation is introduced as $P_{\text {Load }}(t)=$ $P_{\mathrm{PV}}(t)+P_{\text {Grid }}(t)+P_{\text {Batt }}(t)$, where $P_{\text {Load }}(t), P_{\mathrm{PV}}(t), P_{\text {Grid }}(t)$ and $P_{\text {Batt }}(t)$ are the power demands from the load, generated by PV and provided by the power grid and ESS, respectively [9]. From this balance equation, the minimisation problem over time $\sum_{t=0}^{\infty}\left\|P_{\text {Grid }}(t)\right\|_{2}^{2}$ is converted into the minimisation of $\sum_{t=0}^{\infty}\left\|P_{\text {Load }}(t)-P_{\mathrm{PV}}(t)-P_{\mathrm{Batt}}(t)\right\|_{2}^{2}$. The problem is simplified using some assumptions that do not compromise the effectiveness of the control scheme.

The assumptions are 1) a simplified model for the ESS in the form of an integrator is adopted, hence the charge and discharge processes are considered to be identical, 2) the estimation of $P_{\mathrm{PV}}$ is provided by an arbitrary external system, for instance weather forecasts or additional forecasting algorithms, and 3 ) the power to be consumed by the load $P_{\text {Load }}$ is known. In a real case, a forecasting scheme for $P_{\text {Load }}$ should also be adopted, but in order to reduce the number of variables at this stage we assume the power generated by PV is the only variable that is estimated.

The EMS control scheme described in the following section is based on the sampling of PV output power and load demand every $T_{s}$ hour and has a prediction horizon of four hours. For the sake of notational simplicity $t+k$ is used to denote $t+k T_{s}$ to indicate the sampling instance of the $k^{\text {th }}$ sample, where $T_{s}$ is the sampling time. Furthermore, due to the difference between the actual, $P_{\mathrm{PV}}^{\mathrm{Real}}(t)$, and predicted, $P_{\mathrm{PV}}(t)$, power, as explained in section [ it is assumed that for an unknown time-varying value $0 \leq \alpha_{t}^{*} \leq 1$, these powers are related by $P_{\mathrm{PV}}^{\mathrm{Real}}(t)=\alpha_{t}^{*} P_{\mathrm{PV}}(t)$.

\section{B. Notation}

For any variable, $(\cdot)^{\min }$ and $(\cdot)^{\max }$ denote its lower and upper bound respectively. $\mathbb{I}_{i, j}$ represents the set of integers from $i$ to $j$ where $i<j$, i.e., $\mathbb{I}_{i, j}=\{i, i+1, \ldots, j-1, j\}$.

\section{Control Strategy}

A control scheme is proposed to compensate for the error between the predicted PV power and actual output power. The scheme is based on an MPC, which requires numerical optimisation solvers running in real-time, or at least sufficiently fast to be considered as real-time. Unlike standard MPC formulations, the reference trajectory is unknown in advance, hence asymptotic stability is not meaningful in general [10]. Therefore, the focus of the scheme is on the regulation of
$P_{\text {Batt }}(k)$ with respect to the prediction of power generated from $\mathrm{PV}$ and consumed by load, which are denoted by $P_{\mathrm{PV}}(k)$ and $P_{\text {Load }}(k)$.

1) Existing approach: The existing state-of-the-art approach would consider the following optimisation problem:

$$
\begin{aligned}
& \mathbf{P}_{\text {Batt }}\left(t, \mathrm{SOC}_{t}, \mathbf{P}_{\mathrm{PV}}, \mathbf{P}_{\text {Load }}\right)= \\
& \arg \min _{\mathbf{P}_{\text {Batt }}} \sum_{k=t}^{t+N_{h}-1}\left\|P_{\text {Load }}(k)-P_{\mathrm{PV}}(k)-P_{\text {Batt }}(k)\right\|_{2}^{2} \\
& \text { subject to the following constraints } \\
& \operatorname{SOC}(k+1)=\operatorname{SOC}(k)-\frac{T_{s}}{Q_{\text {max }} V_{\text {Batt }}} P_{\text {Batt }}(k), \\
& \operatorname{SOC}(t)=\operatorname{SOC}_{t} \text {, } \\
& \mathrm{SOC}^{\min } \leq \mathrm{SOC}(k) \leq \mathrm{SOC}^{\max }, \\
& P_{\text {Batt }}^{\min } \leq P_{\text {Batt }}(k) \leq P_{\text {Batt }}^{\max }, k \in \mathbb{I}_{t: t+N-1} \text {, }
\end{aligned}
$$

in which $\mathbf{P}_{\text {Batt }}(\cdot)$ is a control sequence over $t$ to $t+N_{h}-1$, so that $\mathbf{P}_{\text {Batt }}=\left(P_{\text {Batt }}(t), P_{\text {Batt }}(t+1), \ldots, P_{\text {Batt }}\left(t+N_{h}-1\right)\right)$, $S O C(k)$ is the present state of charge (SOC) of the battery, $Q_{\max }$ is its nominal current capacity, and $V_{\mathrm{Batt}}$ is the battery voltage. At each time step, the first element of (1), $P_{\text {Batt }}(t)$, is selected and the same procedure is repeated at the next time step.

2) Proposed approach: The main idea of using a scaling factor for improving the performance of the scheme in (1) is presented in the following paragraphs. In order to compensate for the difference between measured, $P_{\mathrm{PV}}^{\mathrm{Real}}$, and previously estimated PV power, $P_{\mathrm{PV}}$, the objective function (1a) is replaced by the following optimisation problem (2).

$$
\begin{aligned}
& \mathbf{P}_{\text {Batt }}\left(t, \mathrm{SOC}_{t}, \mathbf{P}_{\mathrm{PV}}, \mathbf{P}_{\text {Load }}\right)= \\
& \arg \min _{\mathbf{P}_{\text {Batt }}} \sum_{k=t}^{t+N_{h}-1}\left\|P_{\text {Load }}(k)-\alpha_{t} P_{\mathrm{PV}}(k)-P_{\text {Batt }}(k)\right\|_{2}^{2}
\end{aligned}
$$

At time $t$, the scaling factor $0 \leq \alpha_{t} \leq 1$ is determined by the ratio of the summation of $P_{\mathrm{PV}}(t)$ and $P_{\mathrm{PV}}^{\mathrm{Real}}(t)$ over the current and the last sampling time so that

$$
\alpha_{t}=\frac{P_{\mathrm{PV}}^{\mathrm{Real}}(t)+P_{\mathrm{PV}}^{\mathrm{Real}}(t-1)}{P_{\mathrm{PV}}(t)+P_{\mathrm{PV}}(t-1)} .
$$

Note that $\alpha_{t}$ is assigned with the value of 1 when the denominator of (3), $P_{\mathrm{PV}}(t)+P_{\mathrm{PV}}(t-1)$, is equal to zero, in which case the ratio cannot be defined 1 Moreover, this optimisation scheme, (2), is suitable for compensating the mean or persistent differences in the real and predicted output powers. This is because once the factor is evaluated with (3), it is assumed to be a constant for the duration of the sampling time. Therefore, the MPC scheme considers $\alpha$ as a parameter over the length of prediction horizon.

It can be seen that the basic structure of the proposed scheme is close to that of a typical MPC scheme. The main

\footnotetext{
${ }^{1}$ Note that this control strategy may result in violation of input or state constraints due to the difference between the estimated and the real future data. For instance, $P_{\text {Batt }}(t)$ may be assigned with a value greater than the difference from the power of load and PV. The control policy, however, is only used to schedule power allocations between the components of the microgrid, so this violation is absorbed by the power grid.
} 
difference is the formulation of the optimisation problem, (2), using the scaling factor, $\alpha_{t}$. Moreover, the factor has similar characteristics to that of an adjustable lowpass filter, which can be designed to give an appropriate sequence of battery output power. When used as a lowpass filter with a high cutoff frequency, the factor improves the dynamic response of the MPC scheme. By contrast, a low cutoff frequency is beneficial to compensate for the average offset in the real and predicted output power, and is being considered in this paper. It should be noted that the maximum cutoff frequency is limited to the sampling frequency of the optimisation problem. The filter design is not discussed further in the paper, but the performance of the system is investigated for two different sampling times.

Fig. 1 presents a visual representation of the EMS, which comprises four different modules: the interface, the Energy Supply Predictor (ESP), the Energy Demand Predictor (EDP) and the control algorithm. The interface performs the data logging of SOC, $P_{\mathrm{Batt}}, P_{\mathrm{Load}}, P_{\mathrm{PV}}$ and $P_{\mathrm{PV}}^{\mathrm{Real}}$. The four software modules exchange information through a pre-defined communication protocol, where the data formats are selected in advance. This modular structure allows the user to implement their preferred prediction schemes into the ESP and EDP. In order to send the commands to the interface, the control algorithm reads the predicted output values of the PV and the load produced by the ESP and EDP modules, respectively, and executes the control scheme.
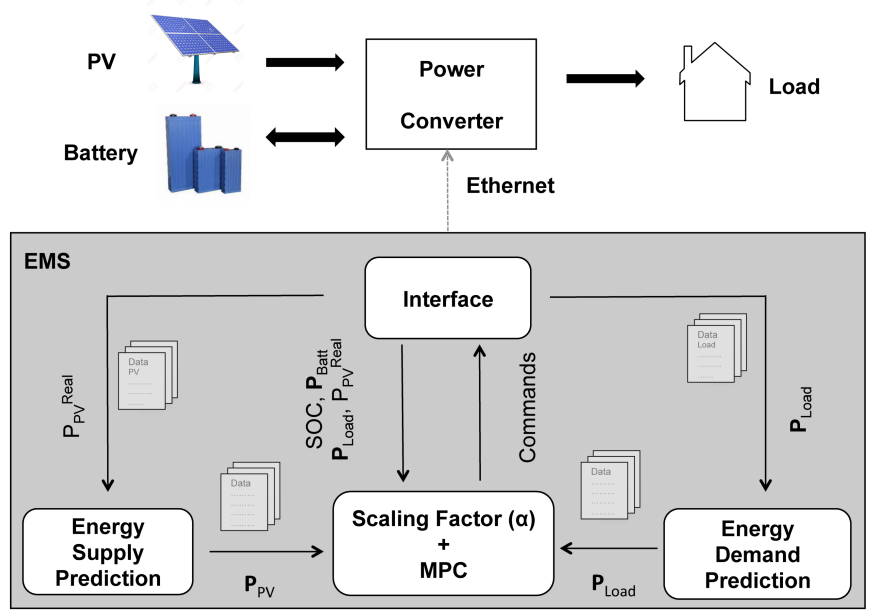

Fig. 1. Energy management system block diagram. The interconnected software modules (rounded rectangles) are linked to the converter via Ethernet connection.

\section{Simulations}

A setup of the microgrid system under consideration is shown in Fig. 2, where a PV panel, battery and grid are connected to a common DC bus, $C_{\mathrm{DC}}$. Each unit employs a power converter (labelled as DC/AC and DC/DC) to convert electrical power from one level to another with regards to frequency, voltage and current. The purpose of each converter is to match loads and supplies of different voltages and frequencies and to provide a stable DC bus. The power ratings of the battery, PV, and grid converters are $5 \mathrm{~kW} / 400 \mathrm{Ah}$,
$10 \mathrm{~kW}$, and $5 \mathrm{~kW}$, respectively. The voltage rating of the battery is $50 \mathrm{~V}$, which is assumed to be constant.

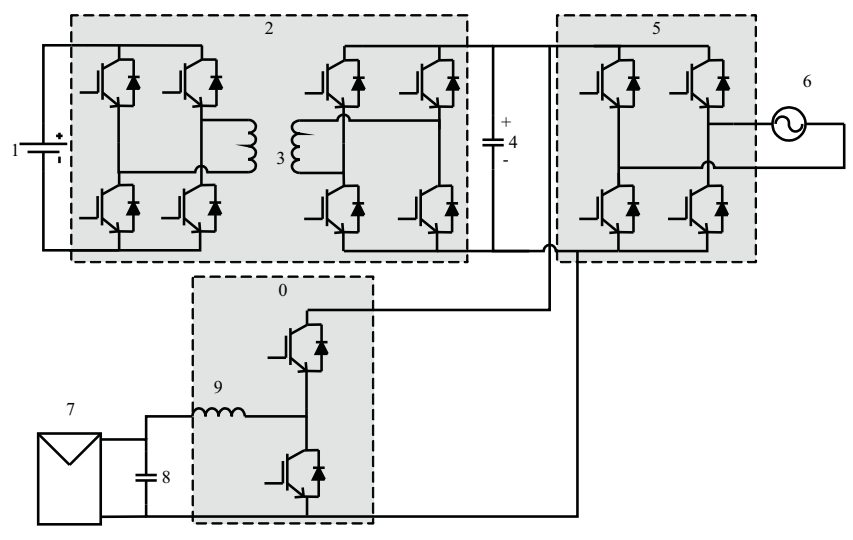

Fig. 2. Schematic diagram of a microgrid system.

The optimisation problem (2) is solved using the quadprog function of the optimisation toolbox in MATLAB. The simulations covers a period of one and a half days. The performance of the system is investigated for two sampling times. Moreover, the performance is evaluated for different values of scaling factor, emulating a range of discrepancies in the values of the real and predicted output powers, and results are presented in the following sections.

\section{A. EMS with a sampling time of 30 minutes}

The MPC scheme is formulated to maximise 'selfconsumption' of locally generated power, in accordance with the explanation given in section III The prediction horizon is set as 4 hours, while the sampling time is 30 minutes, so that $N_{h}=8$ and $T_{s}=0.5$. In order to demonstrate the effectiveness of the concept, the output power of a PV panel is dropped by half, emulating either a fault in some of the converters or shading of some of the panels in a string. For simulations, the output power is reduced by multiplying the PV predicted power by a scaling factor $\alpha$ with a mean value of 0.5 and $20 \%$ variation around the mean value. This variation accounts for the measurements noise in the various sampled signals. However, the information about this factor is not available to the optimisation algorithm, which determines its value using (3). In Fig. 3 this factor is plotted and marked with a symbol $\alpha^{\mathrm{R}}$, while the values determined using (3) are also plotted (symbol $\alpha^{\mathrm{P}}$.) The algorithm resets the $\alpha^{\mathrm{P}}$ to one once the total output power of the PV panel reaches zero, for example, during night time.

For this case, performance of the system is also investigated for the conventional control scheme, (1), which does not account for the difference between the real and predicted output power.

Fig. 4 shows the waveform of the maximum predicted output power of the PV panels and is marked with $\alpha=1$. The waveform associated with $\alpha^{\mathrm{R}}$ shows the predictions of real output power of PV for the choice of this scaling factor. The performance of the proposed scheme is compared to a conventional scheme that uses PV predictions without any 


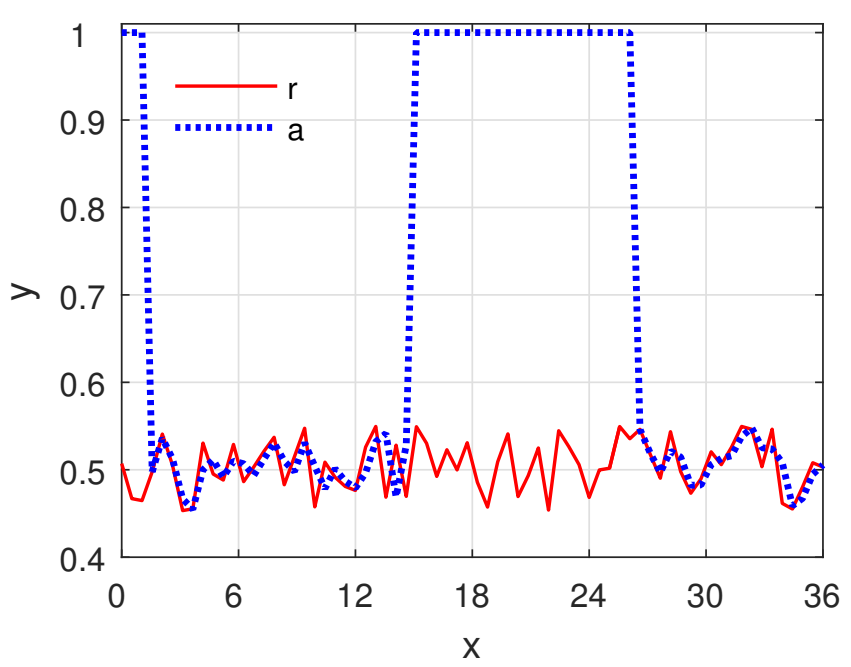

Fig. 3. Actual, $\alpha^{\mathrm{R}}$, and predicted, $\alpha^{\mathrm{P}}$, scaling factor with a sampling time of 30 minutes.

scaling factor, i.e., believes that the PV output power will closely match its predictions. The results with the proposed and conventional schemes are marked with $\alpha^{\mathrm{P}}$ and $\alpha=1$ respectively, in the following discussion. The performance of the system is evaluated using the correct value of the factor, see Fig. 3, and the results associated with this case are marked with $\alpha^{\mathrm{R}}$.

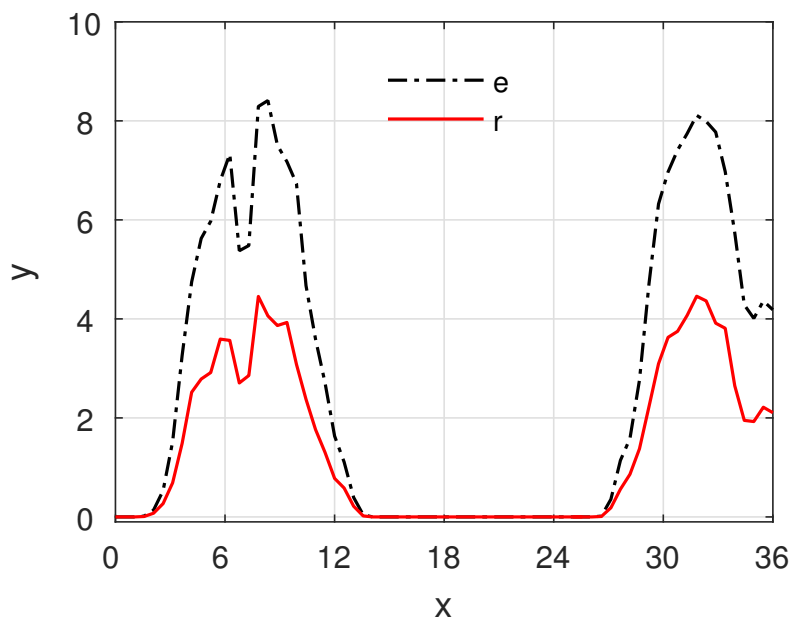

Fig. 4. Output power of PV panels with a sampling time of 30 minutes.

The performance of the system is investigated for a given profile of the power consumed by a load as shown in Fig. 5 and the power taken from the grid is also shown in the same figure. The total energy given by the grid, $E_{\text {Grid }}, 4$ is used to measure the performance of the system.

$$
E_{\text {Grid }}=\frac{1}{2} \int_{t=t_{0}}^{t_{f}}\left(\left|P_{\text {Grid }}(t)\right|+P_{\text {Grid }}(t)\right) \mathrm{d} t,
$$

in which $t_{0}$ is the initial time and $t_{f}$ is the final time for the simulation. The performance index, (4), only considers the power taken from the grid, and excludes the power given to the grid. This index is different from 2, because we are assuming that there is no real benefit in giving power to the grid, whereas there is a monetary cost involved in taking power from the grid. Note that (4) cannot be used as an objective function while solving the quadratic programming due to implementation issues, but it is being used as a measure of the energy consumed by the system.

Over the simulated period, the grid gives a total energy of 91 $\mathrm{kWh}$ in the case of the conventional control scheme $(\alpha=1)$. Here, energy is calculated using (4). This energy drops to 86 $\mathrm{kWh}$ when exact information $\left(\alpha^{\mathrm{R}}\right)$ about the scaling factor is used in (2). With the proposed scheme, the energy is almost identical to the last case with a value of $86 \mathrm{kWh}$. It is apparent that the knowledge of a discrepancy in PV output power helps to minimise the amount of energy exchanged with the grid.

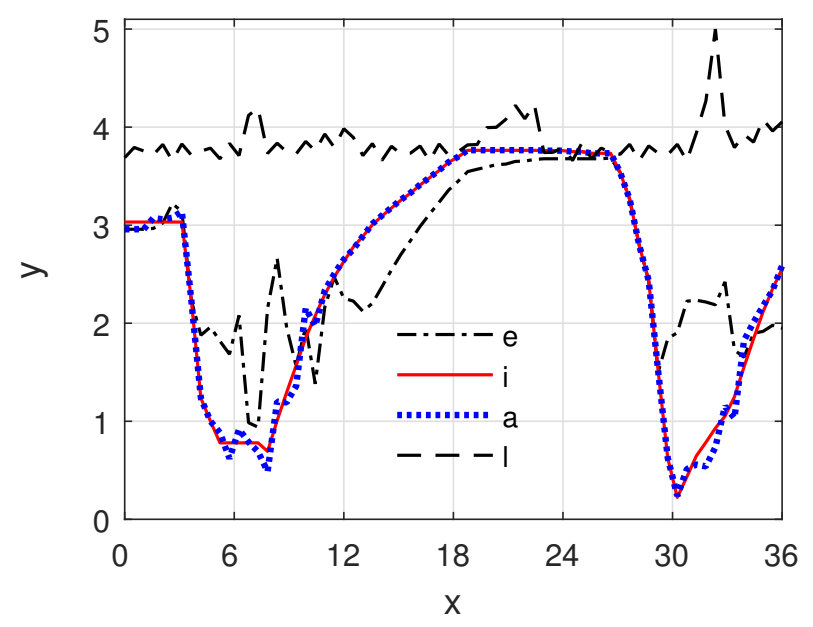

Fig. 5. Load demand and power given by the grid with a sampling time of 30 minutes. The symbol $l$ is used to show power consumed by load and remaining symbols are associated with the power given by the grid.

As shown in Fig. 6, the SOC of the battery shows a significant amount of variation with the conventional control scheme, because the scheme is not aware of any discrepancy between the real and predicted PV power. Therefore, the battery receives power from the grid instead of from the PV panels. This variation is significantly reduced in the proposed scheme and the results closely match the case that uses exact knowledge of the scaling factor. As shown in Fig. 7) the proposed scheme resulted in reduced variation in the power exchanged by the battery.

\section{B. EMS with a sampling time of 15 minutes}

Although the proposed scheme produced satisfactory results, it does not account for the system dynamics with the time period shorter than the previously used sampling time of 30 minutes. Therefore, simulations are carried out to analyse the system performance with the reduced sampling time of 15 minutes. Because the prediction horizon is set to 4 hours, the length of the prediction horizon and sampling time are now set as $N_{h}=16$ and $T_{s}=0.25$, respectively.

The value of the scaling factor determined using (3) is plotted in Fig. 8. Because of the smaller sampling time, the predicted values of the factor are very similar to the actual 


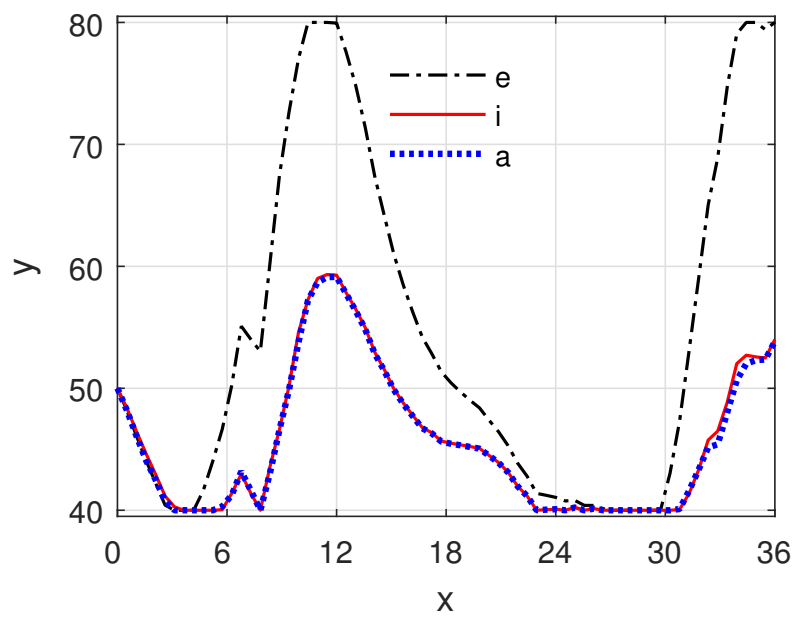

Fig. 6. Battery SOC with a sampling time of 30 minutes.

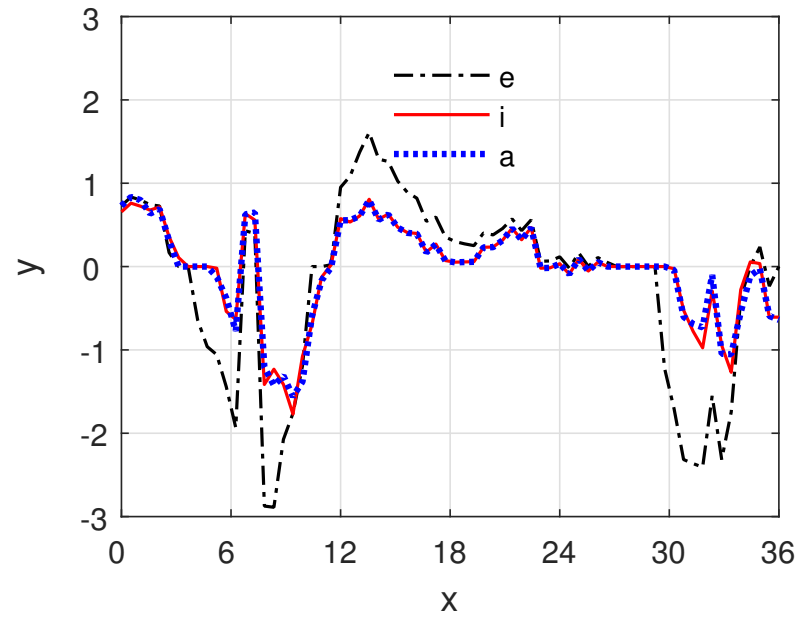

Fig. 7. Battery output power with a sampling time of 30 minutes.

values. As explained before, the algorithm resets the $\alpha^{\mathrm{P}}$ to one once the total output power of the PV panel reaches zero, shown in Fig. 9. In comparison with Fig. 3, the accuracy of the predictions is increased by reducing the sampling time. As expected, the average value of the predictions is not affected by the sampling time. A short sampling time, however, increases variations in the scaling factor, and as a result increases variation in the PV output power shown in Fig. 9

The power given by the grid is shown in Fig. 10 and the overall trend is similar to that of the Fig. 5. However, the conventional scheme, using $\alpha=1$, results in significant variations in the power taken from grid, which are reduced with the proposed scheme. The proposed scheme, $\alpha^{\mathrm{P}}$, again achieves similar waveforms to that of the ideal case, $\alpha^{\mathrm{R}}$.

Since SOC is governed by the average power given or taken by the battery, it is least affected by the small variations in the PV output power shown in Fig. 9. The SOC waveform is very similar to the previous case shown in Fig. 6. However, a small difference in the waveforms can be noticed around the $11^{\text {th }}$ hour, where the battery SOC now saturates to its upper limit of $80 \%$ for a short time period. Unlike SOC, the battery output

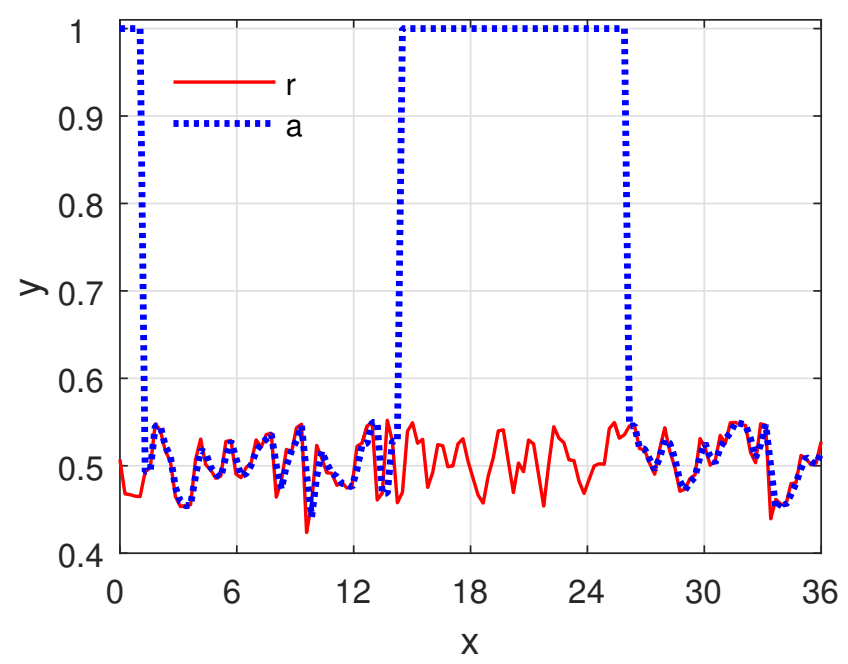

Fig. 8. Actual, $\alpha^{\mathrm{R}}$, and predicted, $\alpha^{\mathrm{P}}$, scaling factor with a sampling time of 15 minutes.

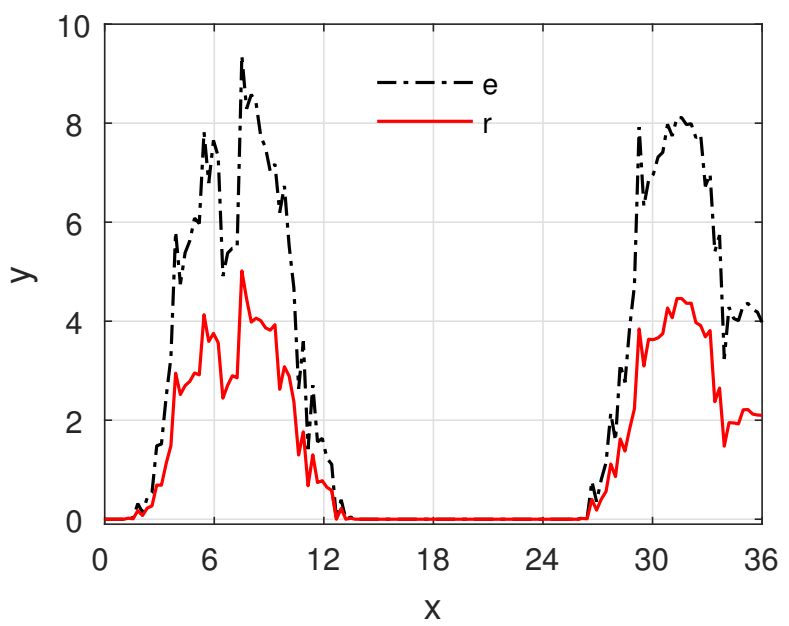

Fig. 9. Output power of PV panels with a sampling time of 15 minutes.

power exhibits increased variations. In comparison to the case with 30 minutes of sampling time, the total energy taken by the battery with the conventional scheme is decreased by $1.5 \%$, the ideal scheme, $\alpha^{\mathrm{R}}$, is increased by $39 \%$, and the proposed scheme, $\alpha^{\mathrm{P}}$, is increased by $39 \%$. Because of the increment in energy, the peak value of the SOC with the purposed scheme, shown in Fig. 11, is slightly increased in comparison to the results shown in Fig. 6

\section{Improvement in performance by altering scaling factor}

In order to further demonstrate the benefits of the proposed scheme, the amount of power given by the grid is analysed over a range of scaling factors. The average value of the scaling factor is varied over the range of $0.2-0.9$, emulating reduction in the PV output power. A scaling factor of $\alpha=0.7$, for example, emulates that PV output power is reduced by $(1-\alpha) \times 100=30 \%$. In this case study, the average value of the scaling factor is varied while keeping the variations of the previously presented cases across its average value. 


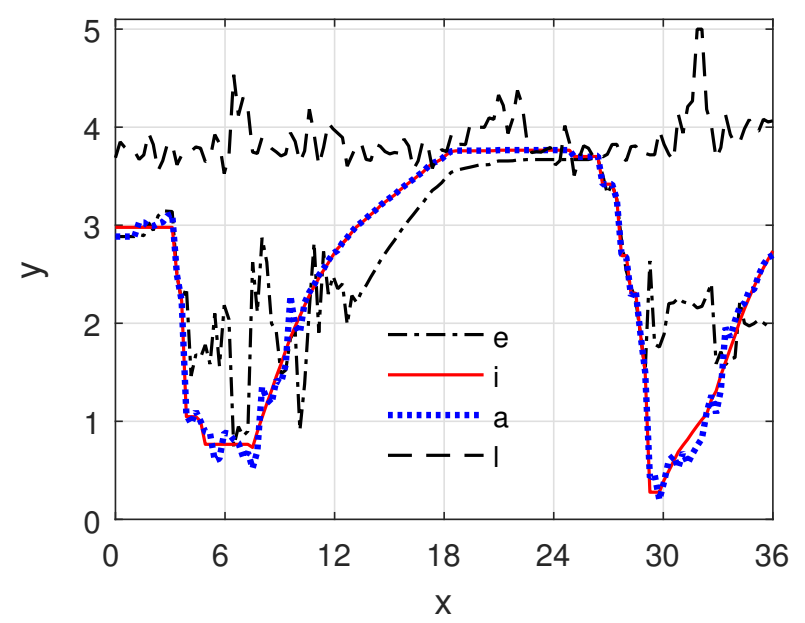

Fig. 10. Load demand and power given by the grid with a sampling time of 15 minutes. The symbol $l$ is used to show power consumed by load and remaining symbols are associated with the power given by the grid.

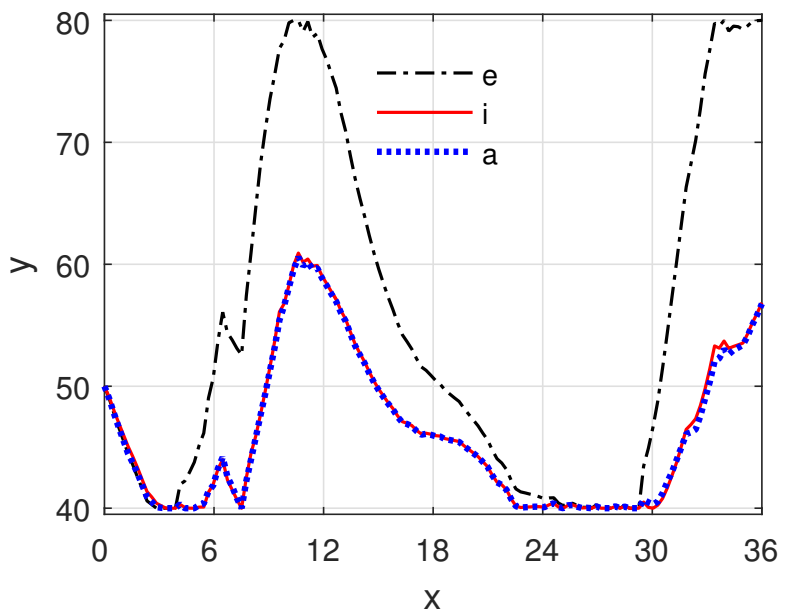

Fig. 11. Battery SOC with a sampling time of 15 minutes.

Fig. 13 shows the waveforms of the total energy given by the grid over the simulated period of 36 hours. Firstly, the simulations are performed for the conventional scheme for both the sampling times and then repeated for the proposed scheme. The microgrid system draws energy from the grid and this energy reduces as $\alpha$ increases. With the $\alpha=0.2$, the grid gives at least $81 \%$ extra energy relative to that with the $\alpha=0.9$. The improvement in performance can be measured by evaluating the difference between the energy without and with the proposed scheme for each value of the factor. The improvement, however, reduces with the increasing $\alpha$ value, because for higher values the system does not allow for further improvements. Consequently, waveforms with the conventional and proposed schemes converge for higher values of the scaling factor. The energy given by the grid with the sampling time of 15 minutes is higher than that of the 30 minutes. This is because of the increased energy intake by the battery with the reduced sampling time, as mentioned in section III-B,

These results indicate an improvement in performance as-

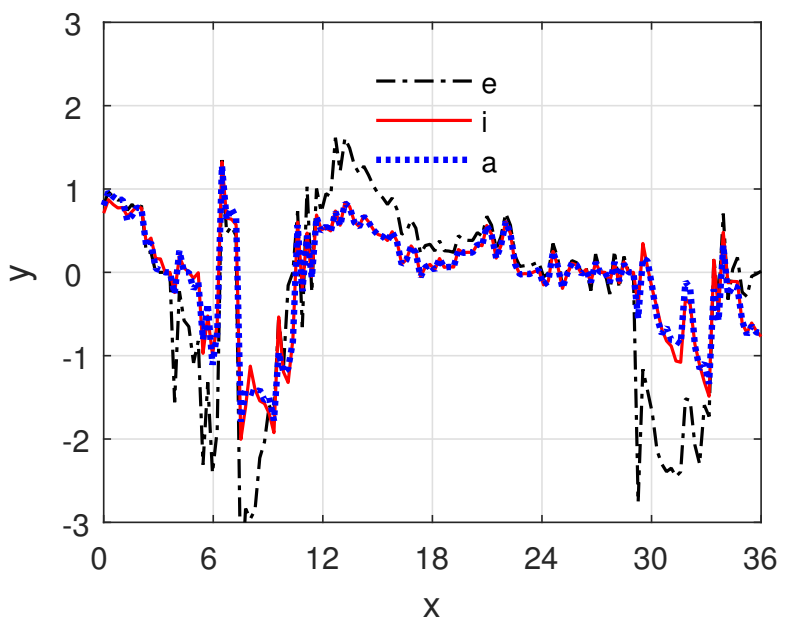

Fig. 12. Battery output power with a sampling time of 15 minutes.

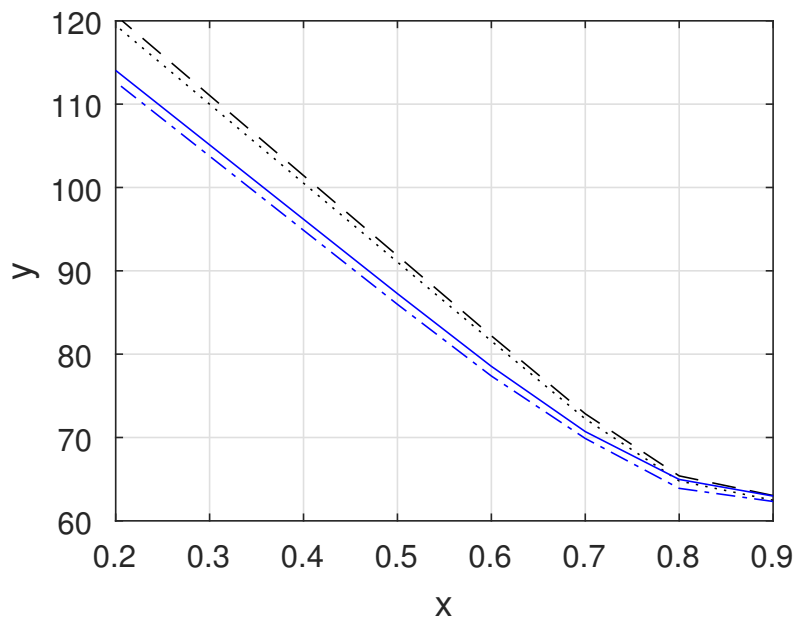

Fig. 13. Energy given by grid for different control scheme and sampling time combinations - conventional scheme with $T_{s}=30$ minutes (dotted line), proposed scheme with $T_{s}=30$ minutes (dashed-dotted line), conventional scheme with $T_{s}=15$ minutes (dashed line), and proposed scheme with $T_{s}=15$ minutes (solid line).

sociated with the proposed scheme. The scaling factor $\alpha$ helps to minimise the power taken from the grid. The value of the scaling factor is evaluated at each sampling instant using a simple division operation as given in (3). The optimisation problem, (2), in then solved using this updated value. Because of the simple division operation, the computational burden of the proposed scheme is similar to that of a typical MPC scheme.

\section{CONCLUSION}

A variant of a model predictive control scheme is proposed to account for the discrepancies in the predicted and actual power output of PV panels. Persistent discrepancies are estimated using a scaling factor whose value is updated at each time step and is used as a parameter for the optimisation problem. The algorithm does not increase the computational burden of a typical MPC scheme and can be implemented on the existing processors running the typical MPC schemes. 
Simulations of a $5 \mathrm{~kW}$ microgrid system are carried out to demonstrate the applicability of proposed method and evaluate improvement in performance associated with it. The results demonstrate that the knowledge of the discrepancy reduce variations in the output power of a battery and also energy taken from grid. The improvement in performance however is a function of the mean value of the scaling factor. A smaller value is associated with a large discrepancy allowing bigger improvement in performance.

\section{ACKNOWLEDGEMENT}

This work is carried out as a part of the project Intelligent Management of Multiple Decentralised Solar/Energy Storage Systems, and is supported by the funding from Korea Institute of Energy Technology Evaluation and Planning (KETEP).

\section{REFERENCES}

[1] C. Hill, M. Such, D. Chen, J. Gonzalez, and W. Grady, "Battery energy storage for enabling integration of distributed solar power generation," IEEE Transactions on Smart Grid, vol. 3, no. 2, pp. 850-857, 2012.

[2] S. Parhizi, H. Lotfi, A. Khodaei, and S. Bahramirad, "State of the art in research on microgrids: A review," IEEE Access, vol. 3, pp. 890-925, 2015.

[3] D. Olivares, A. Mehrizi-Sani, A. Etemadi, C. Canizares, R. Iravani, M. Kazerani, A. Hajimiragha, O. Gomis-Bellmunt, M. Saeedifard, R. Palma-Behnke, G. Jimenez-Estevez, and N. Hatziargyriou, "Trends in microgrid control," IEEE Transactions on Smart Grid, vol. 5, no. 4, pp. 1905-1919, 2014.

[4] S. Teleke, M. Baran, S. Bhattacharya, and A. Huang, "Rule-based control of battery energy storage for dispatching intermittent renewable sources," IEEE Transactions on Sustainable Energy, vol. 1, no. 3, pp. 117-124, 2010.

[5] Y. Riffonneau, S. Bacha, F. Barruel, and S. Ploix, "Optimal power flow management for grid connected PV systems with batteries," IEEE Transactions on Sustainable Energy, vol. 2, no. 3, pp. 309-320, 2011.

[6] D. Q. Mayne, J. B. Rawlings, C. V. Rao, and P. O. M. Scokaert, "Constrained model predictive control: Stability and optimality," vol. 36, no. 6, pp. 789-814, Jun. 2000.

[7] C. Sun, F. Sun, and S. Moura, "Data enabled predictive energy management of a PV-battery smart home nanogrid," in American Control Conference (ACC), 2015, 2015, pp. 1023-1028.

[8] D. Michaelson, H. Mahmood, and J. Jiang, "A predictive energy management strategy with pre-emptive load shedding for an islanded PV-battery microgrid," in Industrial Electronics Society, IECON 2013 - 39th Annual Conference of the IEEE, 2013, pp. 1501-1506.

[9] R. Palma-Behnke, C. Benavides, F. Lanas, B. Severino, L. Reyes, J. Llanos, and D. Saez, "A microgrid energy management system based on the rolling horizon strategy," IEEE Transactions on Smart Grid, vol. 4, no. 2, pp. 996-1006, 2013.

[10] M. A. Stephens, C. Manzie, and Ma. C. Good, "A model predictive control approach to trajectory tracking problems via time-varying level sets of Lyapunov functions," IEEE Transactions on Industrial Informatics vol. 9, no. 2, pp. 808-816, 2013. 